\title{
Probing New Physics in $B \rightarrow \pi K$ Decays
}

\author{
R. Fleischer, ${ }^{a b}$ R. Jaarsma* ${ }^{a}$ E. Malami ${ }^{a}$ and K. K. Vos ${ }^{c}$ \\ ${ }^{a}$ Nikhef \\ Science Park 105, 1098 XG Amsterdam, Netherlands \\ ${ }^{b}$ Department of Physics and Astronomy, Vrije Universiteit Amsterdam \\ NL-1081 HV Amsterdam, Netherlands \\ ${ }^{c}$ Theoretische Physik 1, Naturwissenschaftlich-Technische Fakultät, Universität Siegen \\ D-57068 Siegen, Germany \\ E-mail: Robert.Fleischer@nikhef.nl, Ruben. Jaarsma@nikhef.nl, \\ Eleftheria.Malami@nikhef.nl, keri.vos@uni-siegen.de
}

\begin{abstract}
Over the last two decades the $B \rightarrow \pi K$ decays have received a lot of attention. They are particularly interesting since the main contribution to these decays comes from QCD penguin topologies. Furthermore, electroweak penguin amplitudes give a contribution comparable to the tree topologies. In the past, a discrepancy was found in the correlation between the $\mathrm{CP}$ asymmetries of $B_{d}^{0} \rightarrow \pi^{0} K_{\mathrm{S}}$. We give an up-to-date picture of this situation and consider new constraints, finding that the effect has become larger. An attractive explanation is offered by a modified electroweak penguin sector. We employ an isospin relation between the amplitudes of the $B \rightarrow \pi K$ decays to determine the relevant parameters, making only minimal use of the $S U(3)$ flavour symmetry. Another essential constraint is provided by the mixing-induced CP asymmetry of $B_{d}^{0} \rightarrow \pi^{0} K_{\mathrm{S}}$. The application of our strategy at the next generation of $B$-physics experiments may establish New Physics and reveal new sources of $\mathrm{CP}$ violation in the electroweak penguin sector.
\end{abstract}

Sixth Annual Conference on Large Hadron Collider Physics (LHCP2018)

4-9 June 2018

Bologna, Italy

\footnotetext{
${ }^{*}$ Speaker.
} 


\section{Introduction}

The $B \rightarrow \pi K$ decays have received a lot of attention over the last twenty years ([1,2] and references therein). They are useful for studying electroweak (EW) penguin topologies, which enter in these decays at a level comparable to the tree amplitudes; the CKM matrix element $V_{u b}$ suppresses the latter. Consequently, QCD penguin topologies give the leading contribution.

The $B_{d}^{0} \rightarrow \pi^{0} K_{\mathrm{S}}$ channel is of particular interest because it has a mixing-induced CP asymmetry. In the past, its correlation with the direct $\mathrm{CP}$ asymmetry has revealed a discrepancy, and a modified EW penguin sector was considered as an explanation [2]. We will analyze this correlation using current data and considering new constraints. Moreover, we provide a new strategy to determine the contribution of the EW penguin amplitudes [3].

\section{The $B \rightarrow \pi K$ Decays}

The four $B \rightarrow \pi K$ channels may receive contributions from tree, QCD penguin and EW penguin topologies. The first two enter the amplitudes through the hadronic parameters

$$
r_{\mathrm{c}} e^{i \delta_{\mathrm{c}}} \equiv(\hat{T}+\hat{C}) / P^{\prime}, \quad r e^{i \delta} \equiv\left(\hat{T}-\hat{P}_{t u}\right) / P^{\prime}
$$

Here $\hat{T}(\hat{C})$ are colour-allowed (colour-suppressed) tree contributions, $P^{\prime} \propto P_{t c}$, and $P_{t i}$ denotes the difference between QCD penguins with internal $t$ and $u, c$ quarks. Using the $S U(3)$ flavour symmetry, these parameters can be determined from $B \rightarrow \pi \pi$ data, where EW penguin contributions are tiny $[1,2]$. Including non-factorizable $S U(3)$-breaking corrections of $20 \%$, we obtain [3]

$$
r_{\mathrm{c}} e^{i \delta_{\mathrm{c}}}=(0.17 \pm 0.06) e^{i(1.9 \pm 23.9)^{\circ}}, \quad r e^{i \delta}=(0.09 \pm 0.03) e^{i(28.6 \pm 21.4)^{\circ}} .
$$

In a study of $B_{d, s} \rightarrow h^{+} h^{-}$decays, with $h \in\{\pi, K\}$, we found no sign of anomalously large nonfactorizable $S U$ (3)-breaking effects [4], supporting the assumptions made here.

The EW penguin topologies enter in colour-allowed and colour-suppressed form. The $B_{d}^{0} \rightarrow$ $\pi^{-} K^{+}$and $B^{+} \rightarrow \pi^{+} K^{0}$ channels receive only contributions from the latter, which are expected to be small. However, the $B_{d}^{0} \rightarrow \pi^{0} K^{0}$ and $B^{+} \rightarrow \pi^{0} K^{+}$decays receive contributions from both. These effects can be calculated with help of the $S U(3)$ flavour symmetry, and enter through $[5,1]$ :

$$
q e^{i \phi} e^{i \omega} \equiv-\left(\frac{\hat{P}_{E W}+\hat{P}_{E W}^{C}}{\hat{T}+\hat{C}}\right) \stackrel{S M}{=} \frac{-3}{2 \lambda^{2} R_{b}}\left(\frac{C_{9}+C_{10}}{C_{1}+C_{2}}\right) R_{q}=(0.68 \pm 0.05) R_{q} .
$$

Here, $\phi$ and $\omega$ are CP-violating and CP-conserving phases, respectively. It is a model-independent feature that the latter parameter is small, and it vanishes in the $S U(3)$ limit [6]. Furthermore, $\hat{P}_{E W}^{(C)}$ are the colour-allowed (colour-suppressed) EW penguin topologies, the $C_{i}$ are short-distance coefficients, $\lambda \equiv\left|V_{u s}\right|=0.22$ is the Wolfenstein parameter, and $R_{b}$ is the usual side of the unitarity triangle (UT). The parameter $R_{q}=1.0 \pm 0.3$ allows for $S U$ (3)-breaking corrections. A precision of $5 \%$ is achievable in the future thanks to expected progress in lattice QCD calculations [2].

Experimentally, we have acces to branching ratios $\mathscr{B}^{f}$ and direct $\mathrm{CP}$ asymmetries $A_{\mathrm{CP}}^{f}$ for all four $B \rightarrow \pi K$ channels. Moreover, in the case of the $B_{d}^{0} \rightarrow \pi^{0} K_{\mathrm{S}}$ mode, we have also a mixinginduced CP asymmetry $S_{\mathrm{CP}}^{f}$. The direct CP asymmetries are all proportional to $r_{(\mathrm{c})} \sin \delta_{(\mathrm{c})}$. Consequently, they take values at the $10 \%$ level due to the smallness of these parameters, which are given 
in Eq. (2.2). Furthermore, they enter the following sum rule [7, 3]:

$$
\Delta_{\mathrm{SR}} \equiv\left[A_{\mathrm{CP}}^{\pi^{+} K^{0}} \frac{\mathscr{B}^{\pi^{+} K^{0}}}{\mathscr{B} \pi^{-} K^{+}}-A_{\mathrm{CP}}^{\pi^{0} K^{+}} \frac{2 \mathscr{B} \pi^{0} K^{+}}{\mathscr{B} \pi^{-} K^{+}}\right] \frac{\tau_{B_{d}}}{\tau_{B^{ \pm}}}+A_{\mathrm{CP}}^{\pi^{-} K^{+}}-A_{\mathrm{CP}}^{\pi^{0} K^{0}} \frac{2 \mathscr{B}^{\pi^{0} K^{0}}}{\mathscr{B}^{\pi^{-} K^{+}}}=0+\mathscr{O}\left(r_{(c)}^{2}\right),
$$

which is satisfied experimentally at the $1 \sigma$ level [8]. The $\pi^{0} K^{0}$ final state is difficult to measure for LHCb, so that we have a large uncertainty of $A_{\mathrm{CP}}^{\pi^{0} K^{0}}$. However, using Eq. (2.4) we can predict [3]:

$$
A_{\mathrm{CP}}^{\pi^{0} K^{0}}=-0.14 \pm 0.03 \text {. }
$$

The mixing-induced CP asymmetry is defined through the time-dependent rate asymmetry:

$$
\frac{\Gamma\left(\bar{B}_{d}^{0}(t) \rightarrow \pi^{0} K_{\mathrm{S}}\right)-\Gamma\left(B_{d}^{0}(t) \rightarrow \pi^{0} K_{\mathrm{S}}\right)}{\Gamma\left(\bar{B}_{d}^{0}(t) \rightarrow \pi^{0} K_{\mathrm{S}}\right)+\Gamma\left(B_{d}^{0}(t) \rightarrow \pi^{0} K_{\mathrm{S}}\right)}=A_{\mathrm{CP}}^{\pi^{0} K_{\mathrm{S}}} \cos \left(\Delta M_{d} t\right)+S_{\mathrm{CP}}^{\pi^{0} K_{\mathrm{S}}} \sin \left(\Delta M_{d} t\right) .
$$

Here, $\Delta M_{d}$ is the mass difference between the two $B_{d}$ mass eigenstates. In can be expressed in terms of the direct CP asymmetry, as well as $\phi_{00} \equiv \arg \left(\overline{\mathrm{A}}_{00} \mathrm{~A}_{00}^{*}\right)$, the angle between the decay amplitude $A_{00} \equiv A\left(B_{d}^{0} \rightarrow \pi^{0} K^{0}\right)$ and its CP-conjugate $\bar{A}_{00}$ [2]:

$$
S_{\mathrm{CP}}^{\pi^{0} K_{\mathrm{S}}}=\sin \left(\phi_{d}-\phi_{00}\right) \sqrt{1-\left(A_{\mathrm{CP}}^{\pi^{0} K_{\mathrm{S}}}\right)^{2}}
$$

where also the CP-violating $B_{d}^{0}-\bar{B}_{d}^{0}$ mixing phase $\phi_{d}=(43.2 \pm 1.8)^{\circ}$ enters. Using the hadronic parameters in Eq. (2.1), we find [3]

$$
\tan \phi_{00}=2\left(r \cos \delta-r_{\mathrm{c}} \cos \delta_{\mathrm{c}}\right) \sin \gamma+2 r_{\mathrm{c}}\left(\cos \delta_{\mathrm{c}}-2 \tilde{a}_{\mathrm{C}} / 3\right) q \sin \phi+\mathscr{O}\left(r_{(\mathrm{c})}^{2}\right),
$$

where the small colour-suppressed EW penguin amplitudes enter through $\tilde{a}_{\mathrm{C}} \equiv a_{\mathrm{C}} \cos \left(\Delta_{\mathrm{C}}+\delta_{\mathrm{c}}\right)$.

\section{The CP Asymmetries of $B_{d}^{0} \rightarrow \pi^{0} K_{\mathrm{S}}$}

The $B \rightarrow \pi K$ decay amplitudes are related by isospin, yielding the following relation $[1,2]$ :

$$
\sqrt{2} A\left(B_{d}^{0} \rightarrow \pi^{0} K^{0}\right)+A\left(B_{d}^{0} \rightarrow \pi^{-} K^{+}\right)=\sqrt{2} A\left(B^{+} \rightarrow \pi^{0} K^{+}\right)+A\left(B^{+} \rightarrow \pi^{+} K^{0}\right) \equiv 3 A_{3 / 2} .
$$

The parameter $3 A_{3 / 2} \equiv 3\left|A_{3 / 2}\right| e^{i \phi_{3 / 2}}$ is given by

$$
3 A_{3 / 2}=-(\hat{T}+\hat{C}) e^{i \gamma}+\left(\hat{P}_{E W}+\hat{P}_{E W}^{\mathrm{C}}\right)=-(\hat{T}+\hat{C})\left(e^{i \gamma}-q e^{i \phi} e^{i \omega}\right),
$$

which is an isospin $I=3 / 2$ amplitude. The phase $\gamma=(70 \pm 7)^{\circ}$ is the usual UT angle. The normalization $|\hat{T}+\hat{C}|$ can be determined from the $S U(3)$ relation [9]

$$
|\hat{T}+\hat{C}|=R_{T+C}\left|V_{u s} / V_{u d}\right| \sqrt{2}\left|A\left(B^{+} \rightarrow \pi^{+} \pi^{0}\right)\right|,
$$

where the $V_{u i}$ are elements of the CKM matrix. Within factorization, we have $R_{T+C}=f_{K} / f_{\pi}=$ $1.1928 \pm 0.0026$ [8]. We will allow for non-factorizable effects of up to $100 \%$ of the leading contribution in factorization, yielding $R_{T+C}=1.2 \pm 0.2[2,10]$. Progress in lattice QCD calculations will allow for an improvement of the precision with one order of magnitude [2]. 

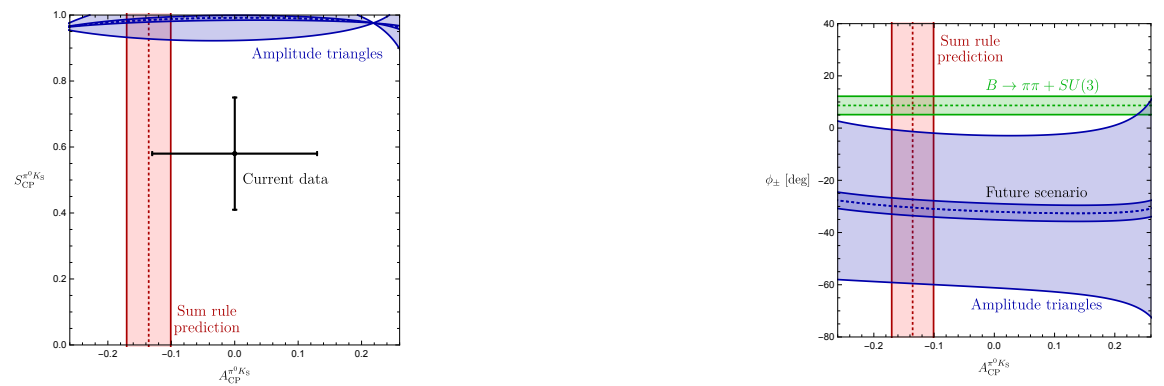

Figure 1: The left panel shows the correlation between the CP asymmetries of $B_{d}^{0} \rightarrow \pi^{0} K_{\mathrm{S}}$ as derived from the amplitude triangles whereas the right panel gives the angle $\phi_{ \pm}$as a function of $A_{\mathrm{CP}}^{\pi^{0} K_{\mathrm{S}}}$.

The expression in Eq. (2.7) describes a correlation between the CP asymmetries of $B_{d}^{0} \rightarrow \pi^{0} K_{\mathrm{S}}$. In order to utilize this relation, we require the angle $\phi_{00}$. It may be determined from Eq. (2.8) using the values of the parameters in Eqs. (2.2) and (2.3). However, the cleanest way is to use the isospin relation in Eqs. (3.1) and (3.2) [2]. Particularly, they describe amplitude triangles in the complex plane, and the angle $\phi_{00}$ can be extracted from the triangles corresponding to the neutral decays. Here, no topologies have to be neglected, and the $S U$ (3) flavour symmetry enters only through $R_{T+C}$ in Eq. (3.3) and $R_{q}$ in Eq. (2.3). There is a fourfold ambiguity in the determination of $\phi_{00}$, which can be resolved using data for the neutral $B \rightarrow \pi K$ decays [2, 3].

Finally, we find the correlation in the left panel of Fig. 1 [3]. Compared to previous work [2], the uncertainty of the contour has been reduced, in particular due to more precise measurements of $\gamma$. The narrow band shows a future scenario where we have assumed perfect measurements as well as more precise determinations of $R_{q}$ and $R_{T+C}$ [2]. We've added a cross denoting the current experimental situation [8], and the red band corresponds to the prediction for $A_{\mathrm{CP}}^{\pi^{0} K_{\mathrm{S}}}$ from the sum rule, given in Eq. (2.5). We note that there is a discrepancy between the contour from the amplitude triangles and the data point at the $2.5 \sigma$ level.

The angle $\phi_{ \pm} \equiv \arg \left(\overline{\mathrm{A}}_{ \pm} \mathrm{A}_{ \pm}^{*}\right)$ between the decay amplitude $A_{ \pm} \equiv A\left(B_{d}^{0} \rightarrow \pi^{-} K^{+}\right)$and its CPconjugate $\bar{A}_{ \pm}$can also be determined from the amplitude triangles. On the other hand, this parameter can be calculated using the hadronic parameters in Eq. (2.2). In particular, we obtain a simple expression for $\phi=0^{\circ}$, as we have in the SM, given by $\left.\phi_{ \pm}\right|_{\phi=0}=2 r \cos \delta \sin \gamma+\mathscr{O}\left(r^{2}\right)=(8.7 \pm 3.5)^{\circ}$. This yields a new constraint [3], shown in the right panel of Fig. 1, where we have also added the future scenario. Again we note a discrepancy, which shows that the correlation from the amplitude triangles itself is in tension with the SM. The situation in Fig. 1 could be resolved if $\mathscr{B} \pi^{0} K^{0}$ goes down by about $2.5 \sigma$ while $S_{\mathrm{CP}}^{\pi^{0} K_{\mathrm{S}}}$ goes up by about $1 \sigma$. Alternatively, this may be a sign of NP. In that case, a modified EW penguin sector offers an attractive scenario.

\section{Extraction of the Electroweak Penguin Parameters}

As discussed in Ref. [3], the isospin relation in Eqs. (3.1) and (3.2) can also be applied to put a constraint on the EW penguin parameters $q$ and $\phi$. The method can be implemented for the charged and neutral decays separately. We have to determine the relative orientation of the triangles. In case of the neutral decays, we can use $S_{\mathrm{CP}}^{\pi^{0} K_{\mathrm{S}}}$ to determine $\phi_{00}$. As we have currently a large uncertainty of $S_{\mathrm{CP}}^{\pi^{0} K_{\mathrm{S}}}$ [8], we will consider the charged decays. Here we may calculate 

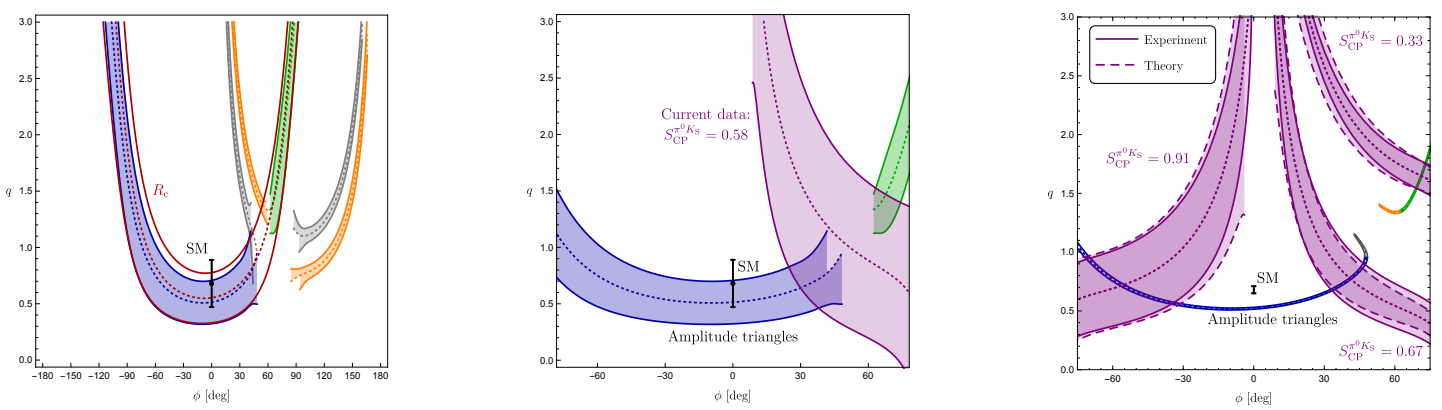

Figure 2: Contours in the $\phi-q$ plane from the amplitude triangles for charged $B \rightarrow \pi K$ data. The left and middle panels correspond to current data, where in the middle panel also the contour from $S_{\mathrm{CP}}^{\pi^{0} K_{\mathrm{S}}}$ was added. The right panel shows future scenarios for measurements of $S_{\mathrm{CP}}^{\pi^{0} K_{\mathrm{S}}}$ as discussed in the text.

the angle $\phi_{\mathrm{c}} \equiv \operatorname{Arg}\left[\bar{A}_{+0} A_{+0}^{*}\right]=\mathscr{O}\left(1^{\circ}\right)$ between the decay amplitude $A\left(B^{+} \rightarrow \pi^{+} K^{0}\right)$ and its $\mathrm{CP}$ conjugate. We then obtain the contours in the left panel of Fig. 2, where we also show the SM point corresponding to Eq. (2.3). No topologies are neglected to implement this method, and it requires only $S U(3)$ input to calculate $|\hat{T}+\hat{C}|$ in Eq. (3.3).

Further insights can be obtained from ratio $R_{\mathrm{c}} \equiv 2\left(\mathscr{B} \pi^{0} K^{+} / \mathscr{B}^{\pi^{+} K^{0}}\right)=1.09 \pm 0.06$. Expressing it in terms of the hadronic parameters we find $R_{\mathrm{c}}=1-2 r_{\mathrm{c}} \cos \delta_{\mathrm{c}}(\cos \gamma-q \cos \phi)+\mathscr{O}\left(r_{\mathrm{c}}^{2}\right)[1,3]$, yielding another contour in the $\phi-q$ plane. It is shown as the red contour in the left panel of Fig. 2, and agrees remarkably well with the blue and green curves from the amplitude triangles.

In order to pin down the values of the EW penguin parameters, we require another contour. To that end, measurements of $S_{\mathrm{CP}}^{\pi^{0} K_{\mathrm{S}}}$ may be used to determine $\phi_{00}$. Using Eq. (2.8), we may then put another constraint on $q$ and $\phi$. Here we use also the values of the hadronic parameters given in Eq. (2.2). The expression is favourable from the theoretical side since the strong phases enter always with a cosine, giving only small variations with respect to these parameters. Moreover, using data we can also include the small colour-suppressed EW penguin effects, entering Eq. (2.8) through $\tilde{a}_{\mathrm{C}}$, as is discussed in detail in Ref. [3]. For current data, we then obtain the purple contour in the middle panel of Fig. 2. We show also the contours from the amplitude triangles in agreement with the $R_{\mathrm{c}}$ constraint, zooming in on the region around the SM point.

Additionally, we consider three scenarios for future measurements of $S_{\mathrm{CP}}^{\pi^{0} K_{\mathrm{S}}}$. These contours are shown in the right panel of Fig. 2. For the contours from the amplitude triangles we assume a more precisie determination of $R_{T+C}$ as for the future scenario in Fig. 1, along with perfect experimental measurments. For the CP asymmetries of $B_{d}^{0} \rightarrow \pi^{0} K_{\mathrm{S}}$, we have assumed an uncertainty of \pm 0.04 , which can be reached at the end of Belle II [11]. Moreover, we allow for non-factorizable $S U$ (3)-breaking effects of $20 \%$ for the hadronic parameters. The experimental and theoretical error bands are shown separately in Fig. 2 by the solid and dashed lines, respectively, illustrating that the experimental precision can be matched by theory.

\section{Conclusions}

Over the years the $B \rightarrow \pi K$ data have shown puzzling patterns. We have presented a stateof-the-art analysis of the correlation between the CP asymmetries of $B_{d}^{0} \rightarrow \pi^{0} K_{\mathrm{S}}$, and found that a previous tension with the data has become stronger. In addition, we have considered a new 
constraint that shows that the correlation itself is not in agreement with the SM. To explain this pattern, either the data for $B_{d}^{0} \rightarrow \pi^{0} K_{\mathrm{S}}$ have to move to agree with the SM, or NP contributions are at work. Should it be the latter, a modified EW penguin sector is an interesting candidate. A new method to determine the EW penguin parameters with unprecedented precision at future experiments has been presented, which will finally clarify this intriguing situation.

\section{Acknowledgments}

This research has been supported by the Netherlands Organisation for Scientific Research (NWO) and by the Deutsche Forschungsgemeinschaft (DFG), research unit FOR 1873 (QFET).

\section{References}

[1] A. J. Buras, R. Fleischer, S. Recksiegel and F. Schwab, $B \rightarrow \pi \pi$, new physics in $B \rightarrow \pi K$ and implications for rare K and B decays, Phys. Rev. Lett. 92 (2004) 101804 [hep-ph/0312259]; Anatomy of prominent $B$ and $K$ decays and signatures of $C P$ violating new physics in the electroweak penguin sector, Nucl. Phys. B 697 (2004) 133 [hep-ph/ 0402112$].$

[2] R. Fleischer, S. Jäger, D. Pirjol and J. Zupan, Benchmarks for the New-Physics Search through CP Violation in $B^{0} \rightarrow \pi^{0} K_{\mathrm{S}}$, Phys. Rev. D 78 (2008) 111501 [arXiv:0806.2900 [hep-ph] ].

[3] R. Fleischer, R. Jaarsma and K. K. Vos, Towards New Frontiers with $B \rightarrow \pi K$ Decays, arXiv:1712.02323 [hep-ph]; R. Fleischer, R. Jaarsma, E. Malami and K. K. Vos, Exploring $B \rightarrow \pi \pi, \pi K$ Decays at the High-Precision Frontier, arXiv:1806.08783 [hep-ph] .

[4] R. Fleischer, R. Jaarsma and K. K. Vos, New strategy to explore CP violation with $B_{s}^{0} \rightarrow K^{-} K^{+}$, Phys. Rev. D 94 (2016) 113014 [arXiv: 1608.00901 [hep-ph] ]; Towards New Frontiers in the Exploration of Charmless Non-Leptonic B Decays, JHEP 1703 (2017) 055 [arXiv: 1612.07342 [hep-ph] ].

[5] M. Neubert and J. L. Rosner, New bound on $\gamma$ from $B^{ \pm} \rightarrow \pi K$ decays, Phys. Lett. B 441 (1998) 403 [hep-ph/9808493]; Determination of the weak phase $\gamma$ from rate measurements in $B^{ \pm} \rightarrow \pi K, \pi \pi$ decays, Phys. Rev. Lett. 81 (1998) 5076 [hep-ph/9809311].

[6] M. Beneke, G. Buchalla, M. Neubert and C. T. Sachrajda, $Q C D$ factorization in $B \rightarrow \pi K, \pi \pi$ decays and extraction of Wolfenstein parameters, Nucl. Phys. B 606 (2001) 245 [hep-ph/ 0104110 ].

[7] M. Gronau, A Precise sum rule among four $B \rightarrow K \pi$ CP asymmetries, Phys. Lett. B 627 (2005) 82 [hep-ph/ 0508047 ]; M. Gronau and J. L. Rosner, Rate and CP-asymmetry sum rules in $B \rightarrow K \pi$, Phys. Rev. D 74 (2006) 057503 [hep-ph/ 0608040 ].

[8] C. Patrignani et al. [PDG], Review of Particle Physics, Chin. Phys. C 40 (2016) 100001

[9] M. Gronau, J. L. Rosner and D. London, Weak coupling phase from decays of charged B mesons to $\pi K$ and $\pi \pi$, Phys. Rev. Lett. 73 (1994) 21 [hep-ph/9404282].

[10] A. Khodjamirian, T. Mannel and M. Melcher, Flavor SU(3) symmetry in charmless B decays, Phys. Rev. D 68 (2003) 114007 [hep-ph/ 0308297 ].

[11] T. Abe et al. [Belle-II Collaboration], Belle II Technical Design Report, arXiv: 1011.0352 [physics.ins-det]. 